\title{
High Estradiol Differentially Affects the Expression of the Glucose Transporter Type 4 in Pelvic Floor Muscles of Rats
}

\author{
María de los Ángeles Carrasco-Ruiz ${ }^{1,2}$, Laura G. Hernández-Aragón ${ }^{3}$, Jesús Ramsés Chávez-Ríos ${ }^{4}$, Jorge Rodríguez-Antolín ${ }^{1}$, \\ Pablo Pacheco ${ }^{4,5}$, Margarita Martínez-Gómez ${ }^{1,4}$, Estela Cuevas-Romero ${ }^{1}$, Francisco Castelán ${ }^{1,4}$ \\ 'Centro Tlaxcala de Biología de la Conducta, Universidad Autónoma de Tlaxcala, Tlaxcala, México \\ ${ }^{2}$ Instituto de Ciencias y Fisiología, Benemérita Universidad Autónoma de Puebla, Puebla, México \\ ${ }^{3}$ Instituto de Fisiología, Benemérita Universidad Autónoma de Puebla, Puebla, México \\ ${ }^{4}$ Depto. de Biología Celular y Fisiología, Instituto de Investigaciones Biomédicas, Universidad Nacional Autónoma de México, Ciudad de México, México \\ ${ }^{5}$ Instituto de Neuroetología, Universidad Veracruzana, Xalapa, México
}

Purpose: To characterize the relationship between serum estradiol levels and the expression of glucose transporter type 4 (Glut4) in the pubococcygeus and iliococcygeus muscles in female rats.

Methods: The muscles were excised from virgin rats during the metestrus and proestrus stages of the estrous cycle, and from sham and ovariectomized rats implanted with empty or estradiol benzoate-filled capsules. The expression of estrogen receptors (ERs) was inspected in the muscles at metestrus and proestrus. Relative Glut4 expression, glycogen content, and serum glucose levels were measured. Appropriate statistical tests were done to identify significant differences $(\mathrm{P} \leq 0.05)$.

Results: The pubococcygeus and iliococcygeus muscles expressed ER $\alpha$ and ER $\beta$. Glut 4 expression and glycogen content in the pubococcygeus muscle were higher at proestrus than at metestrus. No significant changes were observed in the iliococcygeus muscle. In ovariectomized rats, the administration of estradiol benzoate increased Glut4 expression and glycogen content in the pubococcygeus muscle alone.

Conclusions: High serum estradiol levels increased Glut4 expression and glycogen content in the pubococcygeus muscle, but not in the iliococcygeus muscle.

Keywords: Estradiol; Estrogen receptors; Glycogen; Metabolism; Ovariectomy

- Grant/Fund Support: This study was funded by the Dirección General de Asuntos del Personal Académico (DGAPA-UNAM; IA203617) and the Consejo Nacional de Ciencia y Tecnología of México (CONACyT; 225126).

- Research Ethics: The Institutional Committee for Care and Use of Laboratory Animals of the Instituto de Investigaciones Biomédicas of the Universidad Nacional Autónoma de México approved all experimental procedures involving animal subjects included in the present study (project ID number: 243).

- Conflict of Interest: No potential conflict of interest relevant to this article was reported.

\footnotetext{
- HIGHLIGHTS

- Glut4 expression and glycogen content in the pubococcygeus and iliococcygeus muscles are different in metestrus and proestrus.

- High estradiol levels, as a result of administering estradiol benzoate to ovariectomized rats, are related to greater Glut4 expression and glycogen content in the pubococcygeus muscle, but not in the iliococcygeus muscle.

- Both muscles express the $\alpha$ and $\beta$ subtypes of estrogen receptors.
}

Corresponding author: Francisco Castelán (iD https://orcid.org/0000-0002-9835-3335 Universidad Nacional Autónoma de México, Instituto de Investigaciones Biomédicas, Depto. de Biología Celular y Fisiología, Unidad Foránea Tlaxcala, Carr. Fed. TlaxcalaPuebla km 1.5, s/n, 90070-Tlaxcala, México

E-mail: fcocastelan@iibiomedicas.unam.mx / Tel: +52-555-622-6533 /

Fax: +52-246-462-1557

Submitted: May 18, 2018 / Accepted after revision: August 18, 2018 (c) (1) This is an Open Access article distributed under the terms of the Creative Commons Attribution Non-Commercial License (http://creativecommons.org/licenses/by-nc/4.0/) which permits unrestricted non-commercial use, distribution, and reproduction in any medium, provided the original work is properly cited. 


\section{INTRODUCTION}

Ovarian steroids appear to be relevant for the pelvic floor muscles (PFMs). Given the remarkable changes in estradiol levels across women's lifespan, an understanding of estrogenic actions could be useful for managing some urogynecological dysfunctions. In this regard, intravaginal estrogen therapy can improve the results of PFMs training (PFMT), electrical stimulation, and biofeedback in post-menopausal women with stress urinary incontinence [1].

Female rats are commonly used to research the functional organization of the PFMs [2], such as the pubococcygeus and iliococcygeus muscles $[3,4]$. However, the hormonal milieu (i.e., relationship with the estrous cycle) has been consistently disregarded. Notwithstanding, a few reports have indicated intriguing relationships between estradiol levels and innervation, myofiber morphometry, and carbohydrate metabolism in the pubococcygeus [5-7].

Glucose transporter type 4 (Glut4) mediates up to $90 \%$ of the glucose uptake in adult skeletal muscles [8]. Insulin signaling promotes the translocation of Glut4-containing vesicles to the sarcolemma and facilitates Glut4 expression [9]. Estrogen modulates Glut4-dependent glucose uptake by 2 mechanisms: an acute mechanism, influencing the translocation to the sarcolemma, and a chronic mechanism, regulating the expression of Glut4 $[10,11]$. Once inside myofibers, glycolysis and glycogenesis are the main metabolic fates of glucose. Indeed, high Glut4 expression is directly related to glycogen content in skeletal muscles [12]. Moreover, estradiol levels influence Glut4 expression and glycogen content [12-14]. Importantly, glycogenesis predominates as a response to insulin stimulation with the overexpression of Glut4 in transgenic female rats, contrasting with male rats, in which glycolysis predominates [15].

Given the scarce information connecting estrogens with physiological processes in the PFMs, we evaluated herein the effect of estradiol on Glut4 expression and glycogen content in the pubococcygeus and iliococcygeus muscles of female rats. We first identified the expression of estrogen receptor alpha $(\mathrm{ER} \alpha)$ and estrogen receptor beta (ER $\beta)$ in both muscles because their activation modulates Glut4 expression in hindlimb muscles [10]. Second, a preliminary experiment was done to analyze variation in Glut4 expression and glycogen content at metestrus and proestrus, the stages of the estrus cycle in which serum estradiol levels are low and high, respectively [16]. Finally, we performed a second experiment to identify the direct in- fluence of estradiol on Glut4 expression and glycogen content in sham and ovariectomized rats that were or were not administered estradiol benzoate (EB).

\section{MATERIALS AND METHODS}

\section{Animals and Experimental Procedures}

Wistar female rats were housed in individual polypropylene cages ( $37 \mathrm{~cm} \times 27 \mathrm{~cm} \times 16 \mathrm{~cm}$ ) and maintained on a 12-hour light/dark cycle (lights on at $8: 00 \mathrm{PM}$ ) at $20^{\circ} \mathrm{C} \pm 2{ }^{\circ} \mathrm{C}$ in the vivarium of the Centro Tlaxcala de Biología de la Conducta, Universidad Autónoma de Tlaxcala. They were daily provided with pellet food and had continuous access to water.

The estrous cycle of rats was monitored at the age of 3 months by examining vaginal smears [17]; samples were taken between 9:00 AM and 10:00 AM. ER expression in the pubococcygeus and iliococcygeus muscles was assessed at metestrus ( $M$ group; $\mathrm{n}=3$ ) and proestrus ( $\mathrm{P}$ group; $\mathrm{n}=3$ ) in rats that were sacrificed around 10:30 AM and 11:00 AM, after 3 to 5 regular cycles (4 days each). For the first experiment, Glut4 expression and glycogen content were measured in the pubococcygeus and iliococcygeus muscles of 14 rats that were allocated to the $M(n=7)$ and $P$ groups $(n=7)$. For the second experiment, 12 rats were ovariectomized bilaterally under ketamine $(40 \mathrm{mg} / \mathrm{kg}$, intraperitoneally [i.p.]) and xylazine (5 mg/kg, i.p.) anesthesia following a protocol adapted from Olson and Bruce [18]; the other 6 rats were subjected to sham surgery (the Sh group). After 4 weeks, the ovariectomized rats were implanted with an empty capsule (the OVX group) or a capsule filled with EB (the OVX+EB group). The capsules were made with a Silastic tube (10 mm long, 3.18 mm O.D., 1.98 mm I.D.; Dow Corning Corporation, Midland, MI, USA) sealed with wooden plugs. Females in the Sh group were sacrificed 6 weeks after surgery, during the metestrus stage, while those in the OVX and OVX+ EB groups were sacrificed 2 weeks after implantation surgery. In all cases, an overdose of sodium pentobarbital was used (120 $\mathrm{mg} / \mathrm{kg}$, i.p.).

Blood samples were collected by cardiac puncture and allowed to clot for obtaining sera by centrifugation $(10,000 \mathrm{rpm}$ for 15 minutes). Sera were stored at $-80^{\circ} \mathrm{C}$ until assayed. Bilateral pubococcygeus and iliococcygeus muscles were dissected as described elsewhere [19]. The left muscles were frozen immediately after excision and stored at $-80^{\circ} \mathrm{C}$ until assayed. The wet weight of the uterus was recorded to assess the efficacy of the ovariectomy and EB treatment. Body weight was also recorded 
before surgery, 4 weeks after bilateral ovariectomy, and 2 weeks after capsule implantation.

\section{Antibodies}

Immunopeptides were detected with the following primary antibodies: mouse monoclonal immunoglobulin G (IgG) anti-Glut4 (1:200, cat. sc-53566, Santa Cruz Biotechnology, Santa Cruz, CA, USA), mouse monoclonal IgG antiglyceraldehyde phosphate dehydrogenase (1:500, GAPDH, cat. NB300-328, Novus Biologicals, Littleton, CO, USA), mouse monoclonal IgG anti-ATPase subunit 5A (1:500, cat sc-58613, Santa Cruz Biotechnology), mouse monoclonal IgG anti-ERa (1:200, cat. MA3-310, Thermo Scientific, Waltham, MA, USA), and mouse monoclonal IgG anti-ER $\beta$ (1:200, cat. MA1-23217, Thermo Scientific). The secondary antibody was goat anti-mouse $\operatorname{IgG}$ horseradish perioxidase (1:2,000, cat. sc-2005, Santa Cruz Biotechnology).

\section{Western Blot}

Nuclear protein extracts were prepared as described elsewhere [20] and used to identify ER $\alpha$ and ER $\beta$ expression in muscles. Total protein extracts were prepared to measure Glut4 relative expression as previously reported [21]. Briefly, equal amounts of protein $(50 \mu \mathrm{g}$ for nuclear and $100 \mu \mathrm{g}$ for total protein extracts) were denatured in Laemmli's sample buffer, resolved through $10 \%$ sodium dodecyl sulfate (SDS)-polyacrylamide gel electrophoresis and electro-blotted to nitrocellulose membranes. After staining with Ponceau's Red $(0.3 \%$ in acetic acid $1 \%$, Amresco, Solon, OH, USA), the membranes were soaked in phosphate-buffered saline (PBS), blocked with 5\% dried skim milk diluted in PBS, and incubated, first with the primary antibody (overnight at $4^{\circ} \mathrm{C}$ ), and then with the secondary antibody ( 2 hours at room temperature); antibodies were diluted with $1 \%$ dried skim milk in PBS. Immunoreactive polypeptides were detected using a chemiluminescence kit (West Pico Signal, Thermo Scientific) and exposed to a chemiluminescent-signal analyzer (MyECL, Thermo Scientific). Once finished, membranes were stripped with $100 \mathrm{mM}$ glycine $(\mathrm{pH}, 2.3)$ and $1 \%$ SDS, soaked in PBS, blocked with 5\% dried skim milk diluted in PBS, and incubated with mouse monoclonal anti-GAPDH (for estimations in the $\mathrm{M}$ and $\mathrm{P}$ stages) or anti-ATPase subunit $5 \mathrm{~A}$ (for the $\mathrm{Sh}, \mathrm{OVX}$, and OVX+EB groups) overnight at $4^{\circ} \mathrm{C}$; the GAPDH and ATPase bands were revealed as described above. The expression of Glut 4 was measured by densitometry and normalized against that of GAPDH or ATPase expression per muscle and indicated as arbitrary units. ImageJ software
(National Institutes of Health, Bethesda, MD, USA) was used for the densitometric analysis.

\section{Serum Glucose and Muscle Glycogen Levels}

The concentration of serum glucose was measured with a glucose-oxidase-based kit following the manufacturer's instructions (Stanbio Glucose LiquiColor Oxidase, Ref. 1071, Stanbio Laboratory, Boerne, TX, USA). Serum samples were thawed, mixed with glucose assay buffer $(1: 100, \mathrm{v} / \mathrm{v})$, and incubated for 10 minutes at room temperature. Absorbance was measured at the wavelength of $492 \mathrm{~nm}$. Each sample was measured by duplicate and reported as $\mathrm{mmol} / \mathrm{L}$. Muscle glycogen content was measured by adapting a protocol reported by other authors [22]. Briefly, a portion of each muscle was homogenized in heated $2 \mathrm{M} \mathrm{HCl}$ (acid hydrolysis of glycogen chains releasing glucose molecules) or heated $2 \mathrm{M} \mathrm{NaOH}$ (basic hydrolysis, no glucose release). Samples were heated in boiling water for 1 hour and centrifuged at 10,000 rpm for 5 minutes at room temperature. The supernatant containing the product of hydrolysis was measured using a colorimetric enzymatic method to measure glucose using the kit mentioned above (Stanbio Laboratory). The muscle glycogen content was calculated as the difference between glycogen chains and free glucose and expressed as millimoles of glycosyl units per gram of muscle wet weight.

\section{Serum Estradiol Levels}

Total serum steroids were extracted with diethyl ether (SigmaAldrich, St. Louis, MO, USA) as reported elsewhere [21]. Total serum estradiol levels were then measured with an enzyme immunoassay-based commercial kit following the manufacturer's instructions (item 582251, Cayman Chemical, Ann Arbor, MI, USA). Each sample was measured in duplicate.

\section{Statistical Analysis}

All measured variables are presented as mean \pm standard error of the mean unless otherwise stated. The level of statistical significance was set at $\mathrm{P} \leq 0.05$. The unpaired 2-tailed Student $\mathrm{t}$ test was used to identify significant differences between variables measured at metestrus and proestrus. For the Sh, OVX, and OVX+EB groups, significant differences regarding serum estradiol levels (data were log-transformed for this statistical test), Glut4 expression, and glycogen content were detected using 1-way analysis of variance followed by the uncorrected Fisher least significant difference test. Due to technical difficulties, glycogen content in the iliococcygeus muscle was mea- 
sured only in 4 rats in the Sh and OVX groups, and in 6 rats in the OVX+EB group. All statistical tests were done using the software Prism (GraphPad Software, La Jolla, CA, USA).

\section{RESULTS}

\section{Estrogen Receptor Expression in the Pubococcygeus and Iliococcygeus Muscles}

The presence of a band of $-66 \mathrm{kDa}$ revealed ERa expression in the pubococcygeus and iliococcygeus muscles at metestrus and proestrus (Fig. 1A). Similarly, a band of $-55 \mathrm{kDa}$ revealed ER $\beta$ expression in the pubococcygeus and iliococcygeus muscles of rats at metestrus and proestrus (Fig. 1B).

\section{Experiment 1}

\section{Glut4 expression in the pubococcygeus and iliococcygeus mus- cles}

Glut4 expression (-50 kDa) was observed in the pubococcygeus and iliococcygeus muscles (Fig. 2A). Glut4 expression in the pubococcygeus muscle at proestrus was significantly higher than at metestrus ( $\mathrm{P}=0.015)$ (Fig. 2B, D). For the iliococcygeus muscle, Glut4 expression was not significantly different between both stages of the estrous cycle ( $\mathrm{P}=0.739)$ (Fig. 2C, E).

\section{Glycogen content in the pubococcygeus and iliococcygeus mus- cles and serum glucose levels}

The glycogen content of the pubococcygeus muscle at proestrus

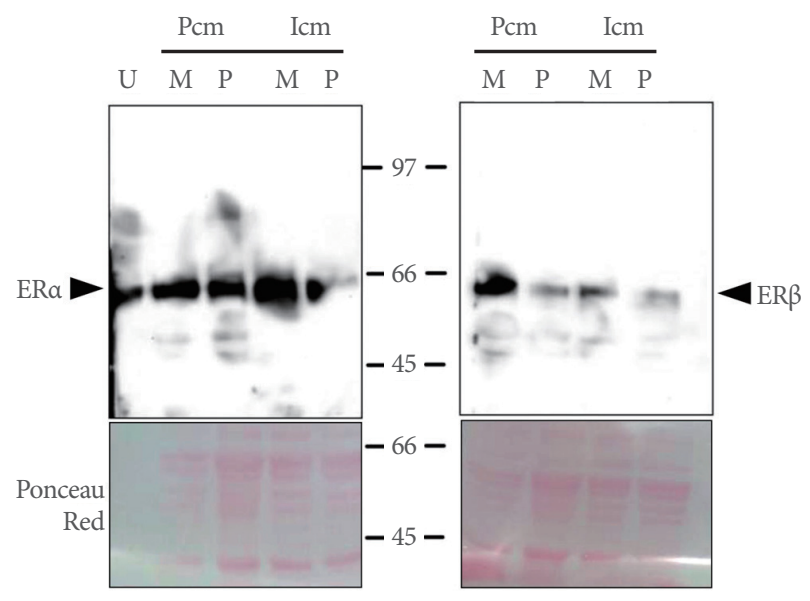

Fig. 1. Expression of ER $\alpha$ and ER $\beta$ in the Pcm and Icm of rats at metestrus $(\mathrm{M})$ and proestrus $(\mathrm{P})$. Nuclear protein extracts from the Pcm and Icm were loaded. Expression of ERa was assessed in uterine extracts $(\mathrm{U})$ as a positive control of ERa expression. Representative photographs of Ponceau's Red-stained membranes are shown. ER, estrogen receptor; Pcm, pubococcygeus muscle; Icm, iliococcygeus muscle.
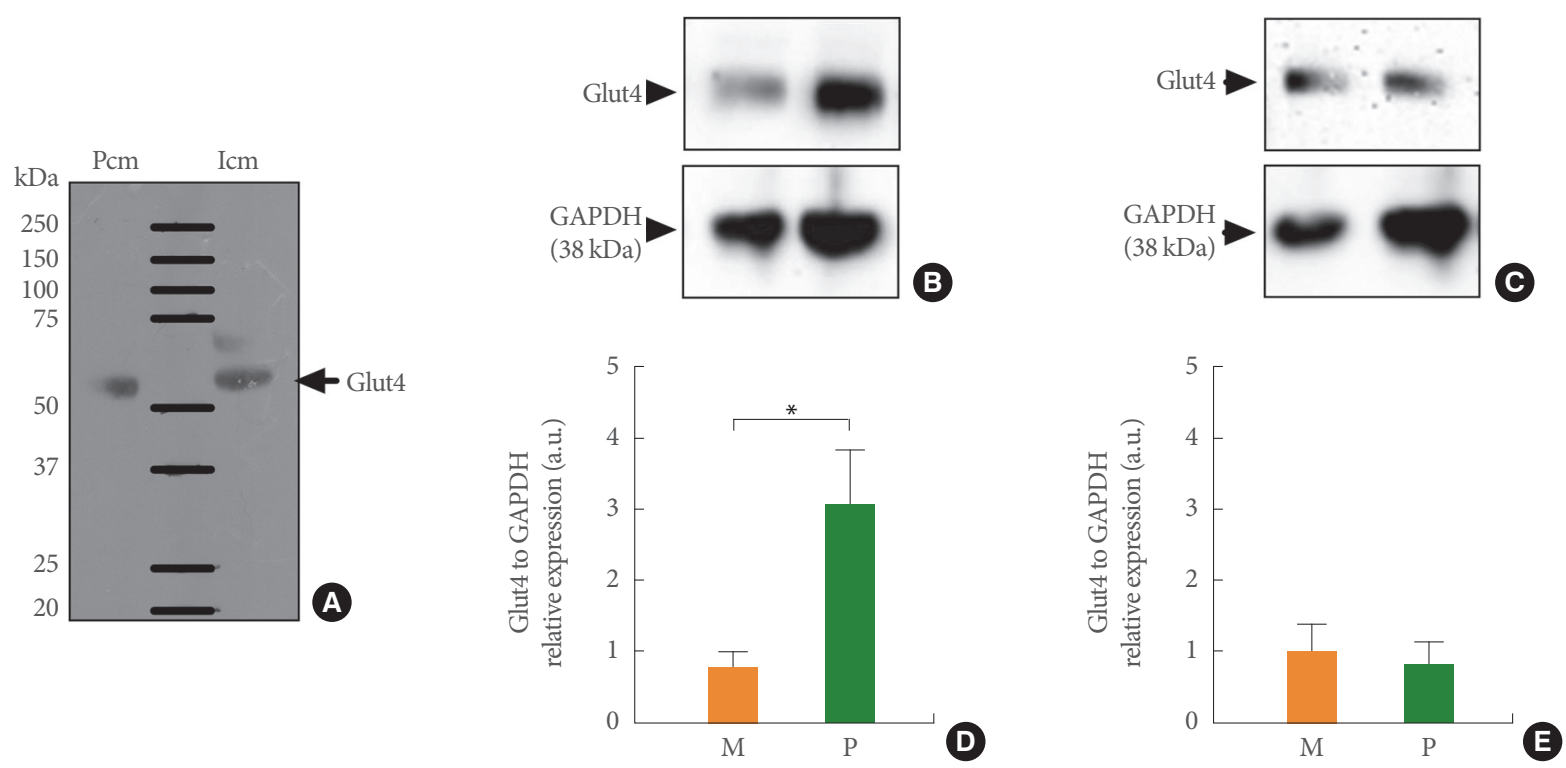

Fig. 2. Glut4 expression in the Pcm and Icm at metestrus (M) and proestrus (P). (A) Representative immunoblot showing Glut4 expression in the Pcm and Icm. Relative expression of Glut4 in the Pcm (B) and Icm (C) is shown as the quotient (arbitrary units [a.u.]) obtained by dividing the density of the Glut4 band by that of the glyceraldehyde phosphate dehydrogenase (GAPDH) band (D, E). Data are means \pm standard error of the mean ( $n=7$ for each group). Two-tailed unpaired t-tests; ${ }^{*} \mathrm{P}<0.05$. Glut 4 , glucose transporter 4 ; Pcm, pubococcygeus muscle; Icm, iliococcygeus muscle. 
was significantly higher than at metestrus $(9.8 \pm 3.7$ vs. $0.96 \pm 0.28$ mmol of glycosyl units per gram of muscle wet weight, $n=7$; $\mathrm{P}=0.035)$. In contrast, the glycogen content of the iliococcygeus at metestrus and proestrus was not significantly different $(3 \pm 2.1$ vs. $1.3 \pm 0.73 \mathrm{mmol}$ of glycosyl units per gram of muscle wet weight, $\mathrm{n}=7 ; \mathrm{P}=0.471)$. However, the concentration of serum glucose at proestrus $(8.5 \pm 0.7 \mathrm{mmol} / \mathrm{L}, \mathrm{n}=7)$ was significantly lower than at metestrus $(12.1 \pm 1 \mathrm{mmol} / \mathrm{L} ; \mathrm{t}=2.943 ; \mathrm{P}=0.012 ; \mathrm{n}=7)$.

\section{Experiment 2}

\section{Bodily measurements}

The body weight at sacrifice was not significantly different $(\mathrm{P}=$ $0.129)$ among the Sh $(249 \pm 12 \mathrm{~g})$, OVX $(287.9 \pm 15.6 \mathrm{~g})$, and $\mathrm{OVX}+\mathrm{EB}(260.4 \pm 10 \mathrm{~g})$ groups. However, 4 weeks after surgery, the body weight gain of the ovariectomized rats was significantly higher than that of the rats subjected to the sham surgery
$(\mathrm{P}=0.003)$ (Fig. 3A). Two weeks after the capsule implantation, the body weight gain was significantly different among the Sh, OVX, and OVX $+\mathrm{EB}$ groups $(\mathrm{P}=0.008)$, and post hoc tests showed that the body weight gain in the OVX group was significantly higher than that in the Sh and OVX+EB groups (Fig. $3 \mathrm{~A})$. The normalized weight of the uterus ( $\mathrm{mg} / \mathrm{g}$ body weight) changed significantly among groups $(\mathrm{P}=0.0059)$, and post hoc tests indicated that a significant reduction only took place in the OVX group, but not in the Sh and OVX+EB groups (Fig. 3B). The concentration of serum estradiol was significantly different among groups $(\mathrm{P}<0.0001)$. Thus, the estradiol concentration significantly decreased in the OVX group in comparison with the Sh group, while a significant increase was found in the $\mathrm{OVX}+\mathrm{EB}$ group in comparison with both the Sh and OVX groups (Fig. 3C).
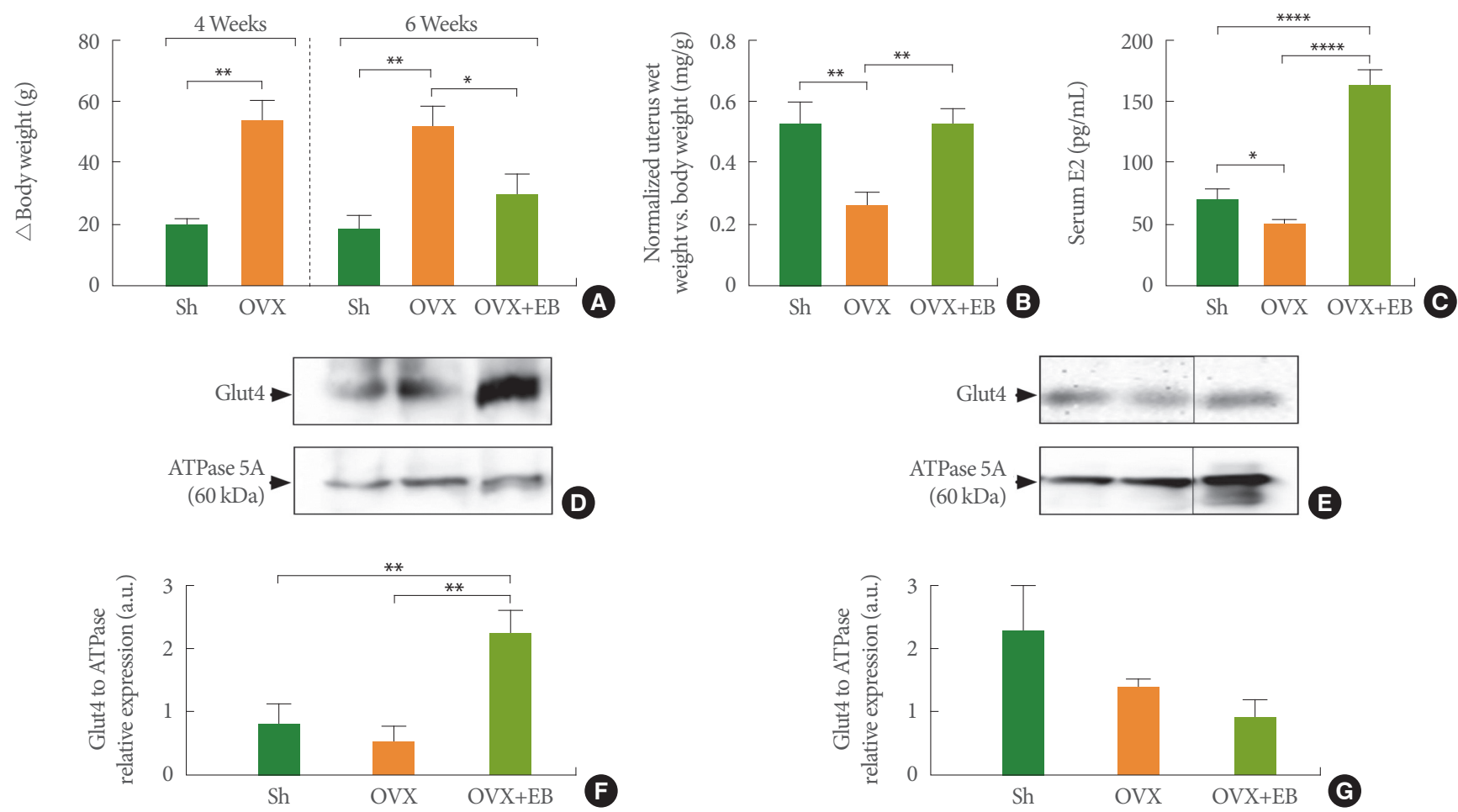

Fig. 3. Glut4 expression in the Pcm and Icm in the Sh, OVX, and OVX+EB groups. (A) Body weight gain of female rats at 4 and 6 weeks (2 weeks after capsule implantation, vertical dashed line) after bilateral ovariectomy. (B) Uterus wet weight at sacrifice day. (C) Serum estradiol levels. Representative immunoblots of Glut4 and ATPase 5A in Pcm (D) and Icm (E); the Icm samples were run in the same gel and a lane was removed for final presentation (note solid line at splice site) because it was not related to the present study. Relative expression of Glut4 in the Pcm (F) and Icm (G) is shown as the quotient (arbitrary units [a.u.]) obtained by dividing the density of the Glut 4 band by that of the ATPase subunit $5 \mathrm{~A}$ (ATPase $5 \mathrm{~A}$ ) band (D, E). Data are means \pm standard error of the mean. ( $\mathrm{n}=6$ for each group). ${ }^{*} \mathrm{P}<0.05$. ${ }^{* *} \mathrm{P}<0.01 .{ }^{* * *} \mathrm{P}<0.0001$. Glut4, glucose transporter $4 ; \mathrm{Pcm}$, pubococcygeus muscle; Icm, iliococcygeus muscle; Sh, sham; OVX, ovariectomized; EB, estradiol benzoate. 


\section{Glut4 expression in muscle and variation in serum estradiol levels}

The expression of Glut4 in the pubococcygeus muscle was significantly different among the groups $(\mathrm{P}=0.003)$ (Fig. 3D, F). Glut4 expression was higher in the OVX+EB group than in the Sh and OVX groups, while no significant difference was detected between the Sh and OVX groups. In contrast, the expression of Glut4 in the iliococcygeus muscle did not significantly differ among the 3 groups $(\mathrm{F}=2.221, \mathrm{P}=0.143)$ (Fig. $3 \mathrm{E}, \mathrm{G})$.

\section{Muscle glycogen and variation in serum estradiol levels}

The glycogen content of the pubococcygeous muscle was significantly different $(\mathrm{P}=0.046)$ among the Sh $(1.2 \pm 0.4 \mathrm{mmol}$ of glycosyl units per gram of muscle wet weight), OVX $(0.96 \pm 0.3$ mmol of glycosyl units per gram of muscle wet weight), and OVX+EB $(2.2 \pm 0.3 \mathrm{mmol}$ of glycosyl units per gram of muscle wet weight) groups, and post hoc tests indicated a significant increase in the glycogen content of the OVX+EB group compared with the Sh $(\mathrm{P}<0.05)$ and OVX groups $(\mathrm{P}<0.05)$. No significant differences were found between the Sh and OVX groups. In contrast, the glycogen content of the iliococcygeus muscle was not significantly different $(\mathrm{P}=0.298)$ among the $\mathrm{Sh}(0.8 \pm 0.5 \mathrm{mmol}$ of glycosyl units per gram of muscle wet weight), OVX $(0.13 \pm 0.04$ mmol of glycosyl units per gram of muscle wet weight), and $\mathrm{OVX}+\mathrm{EB}(0.44 \pm 0.13 \mathrm{mmol}$ of glycosyl units per gram of muscle wet weight) groups. Otherwise, the serum glucose levels did not significantly vary among the groups (Sh: $9.5 \pm 0.7$; OVX: $10.1 \pm$ 0.5; OVX+EB: $8.8 \pm 0.4 \mathrm{mmol} / \mathrm{L} ; \mathrm{P}=0.320$ ).

\section{DISCUSSION}

Considering the positive modulation that high estradiol levels exert on Glut4 expression in hindlimb muscles [14], it seems reasonable to suggest that its rise at proestrus could lead to augmented Glut4 expression in the pubococcygeus muscle. Nevertheless, testosterone and progesterone levels also increase at proestrus $[16,23]$. In this regard, the former hormone could promote augmented Glut4 expression, while the latter could reduce it $[14,24]$. Thus, differences in the relative levels of ovarian steroids may influence Glut4 expression in the pubococcygeus muscle. Although both the pubococcygeus and iliococcygeus muscles express ER $\alpha$ and ER $\beta$, the fact that the iliococcygeus muscle seems to be unresponsive to variation in steroid hormones at metestrus and proestrus may be thus related to the opposing actions initiated by testosterone, progesterone, and estradiol. In any case, further experiments should evaluate the role of the expression of androgen and progesterone receptors in Glut4 expression in the muscles evaluated herein. Such an analysis should include the ER subtypes. Furthermore, serum insulin levels reach their highest levels at proestrus and their lowest levels at diestrus [23]. Although we did not measure insulin levels herein, it could reasonably be expected that the increase in serum insulin levels at proestrus would increase Glut 4 expression similarly in the pubococcygeus and iliococcygeus muscles, but this was not the case.

Bilateral ovariectomy was effective in reducing circulating estradiol, as confirmed by the body weight gain and normalized uterus weight. In contrast, ovariectomized rats implanted with EB capsules had higher estradiol levels. Thus, the present data support the proposal that Glut4 expression in the iliococcygeus muscle is not related to serum estradiol levels because this remained unaltered in the OVX and OVX+EB groups. Such a finding was clearly different from that observed in the pubococcygeus muscle, in which high serum estradiol levels were associated with augmented Glut4 expression. Certainly, musclespecific factors, including MEF2, MyoD, and GEF, directly regulate the transcription of the SLC2A4 gene, which codes for Glut4, while estradiol could act more indirectly [9]. The results herein and those of other studies support the notion that high estradiol levels at proestrus can upregulate Glut4 expression in the pubococcygeus muscle [14,25]. The mechanisms involved in the specific modulation of Glut4 expression in the pubococcygeus and iliococcygeus muscles should be further explored by analyzing transcriptional repression and/or epigenetics triggered by ER activation $[9,25,26]$. In this regard, it is important to consider that ER $\alpha$ activation promotes, while ER $\beta$ activation represses, Glut4 expression in hindlimb muscles [10]. Knowing the ER $\alpha$-to-ER $\beta$ ratio would have been an excellent starting point for an analysis of this issue, but unfortunately we did not measure this ratio herein due to the insufficient number of rats and the primary intention of the study to identify ER expre ssion in the pubococcygeus and iliococcygeus muscles.

Whereas the glycogen content of the pubococcygeus muscle at proestrus was higher than at metestrus, there was no change in the glycogen content of the iliococcygeus between both stages of the estrous cycle. Some studies have demonstrated that higher Glut4 expression was associated with greater glycogen content in skeletal muscles $[12,15]$, which may explain our findings about the pubococcygeus muscle. However, ovarian steroids can influence hindlimb muscle glycogen content, as supported by the fact that estradiol has a positive effect while progesterone has 
a counteracting negative effect [13,27]. Certainly, greater glycogen content could imply a higher rate of synthesis or a slower rate of degradation. In this regard, estradiol restores the glycogen content in the red quadriceps muscle after exercise in ovariectomized rats, while progesterone has no effect [14]. Hence, the present findings from ovariectomized rats treated with EB resemble the findings of high glycogen content in the pubococcygeus at proestrus, supporting the notion that estrogenic actions are involved. In clear contrast, no changes in glycogen content were seen in the iliococcygeus muscle.

The limitations of our present study include the fact that the rats were not fasting before the tissues were excised and that serum insulin levels were not measured. Both of these factors are relevant for understanding the metabolism of glucose in skeletal muscles. However, our data support the proposal that ovarian hormones, and particularly estradiol, may exert a complimentary role in modulating Glut4 expression and glycogen content in the pubococcygeus muscle, but not in the iliococcygeus muscle, as the extent to which circulating insulin levels would influence them is expected to be similar (i.e., higher insulin levels in nonfasting rats are associated with greater Glut4 expression in both muscles). The functional expression of insulin receptors and negative regulators of insulin signaling should therefore be approached for each muscle.

The present findings support the proposal that the pubococcygeus muscle is sensitive to changes in the hormonal milieu due to the estrous cycle. Insights from Glut4 expression and glycogen content in the pubococcygeus muscle point to the involvement of estrogenic actions that should be properly analyzed in further experiments. The apparent insensitivity shown by the iliococcygeus muscle also requires further evaluation, with an emphasis on the expression of particular ER subtypes and other steroid receptors (e.g., androgen and progesterone receptors). As different responses were observed regarding Glut4 expression in each muscle, and since Glut 4 expression is upregulated by insulin and exercise in hindlimb muscles [7], the findings herein could be relevant for understanding the synergistic actions between PFMT and estrogen administration for strengthening the PFMs [1]. Overall, differences regarding Glut4 expression and glycogen content in the pubococcygeus and iliococcygeus muscles could be linked to ovarian steroid levels at proestrus and metestrus. By analyzing these variables in ovariectomized rats according to $\mathrm{EB}$ treatment, the findings herein suggest that high estradiol levels are related to greater Glut4 expression and glycogen content in the pubococcygeus muscle, but not in the iliococcygeus muscle, although both muscles express ERs.

\section{ACKNOWLEDGEMENTS}

The authors thank Laura García Rivera, Guadalupe Citlaly Hernández Hernández, and Shareth Y. Rodríguez Jaimes for the excellent technical assistance provided.

\section{AUTHOR CONTRIBUTION STATEMENT}

- Full access to all the data in the study and takes responsibility for the integrity of the data and the accuracy of the data analysis: $C F$

-Study concept and design: $C F$

- Acquisition of data: CRMA, HALG, JRCR

- Analysis and interpretation of data: CRMA, HALG, JRCR

- Drafting of the manuscript: $C R M A, C F$

- Critical revision of the manuscript for important intellectual content: RAJ, PP, CRE, MGM

- Statistical analysis: $C R M A, C F$

- Obtained funding: $C F$

- Administrative, technical, or material support: $R A J$

- Study supervision: $P P, C R E, M G M, C F$

\section{REFERENCES}

1. Castellani D, Saldutto P, Galica V, Pace G, Biferi D, Paradiso Galatioto G, et al. Low-dose intravaginal estriol and pelvic floor rehabilitation in post-menopausal stress urinary incontinence. Urol Int 2015;95:417-21.

2. Jiang HH, Damaser MS. Animal models of stress urinary incontinence. Handb Exp Pharmacol 2011;(202):45-67.

3. Poortmans A, Wyndaele JJ. Preventing fatigue of fast striated muscles of the pelvic floor and slow striated muscles of the limb by manipulating the on-off time of electric stimulation. Arch Phys Med Rehabil 2002;83:550-4.

4. Poortmans A, Wyndaele JJ. M. levator ani in the rat: does it really lift the anus? Anat Rec 1998;251:20-7.

5. Cuevas E, Camacho M, Alvarado M, Hudson R, Pacheco P. Participation of estradiol and progesterone in the retrograde labeling of pubococcygeus motoneurons of the female rat. Neuroscience 2006; 140:1435-42.

6. Lara-García M, Alvarado M, Cuevas E, Cortés-sol A, Domínguez A, Tovar A, et al. The effects of castration and hormone replacement on the cross-sectional area of pubococcygeus muscle fibers in 
the female rat. Anat Rec (Hoboken) 2011;294:1242-8.

7. Max SR, Knudsen JF. Effect of sex hormones on glucose-6-phosphate dehydrogenase in rat levator ani muscle. Mol Cell Endocrinol 1980;17:111-8.

8. Richter EA, Hargreaves M. Exercise, GLUT4, and skeletal muscle glucose uptake. Physiol Rev 2013;93:993-1017.

9. Im SS, Kwon SK, Kim TH, Kim HI, Ahn YH. Regulation of glucose transporter type 4 isoform gene expression in muscle and adipocytes. IUBMB Life 2007;59:134-45.

10. Barros RP, Gustafsson JÅ. Estrogen receptors and the metabolic network. Cell Metab 2011;14:289-99.

11. Gupte AA, Pownall HJ, Hamilton DJ. Estrogen: an emerging regulator of insulin action and mitochondrial function. J Diabetes Res 2015;2015:916585.

12. Kim YB, Peroni OD, Aschenbach WG, Minokoshi Y, Kotani K, Zisman A, et al. Muscle-specific deletion of the Glut4 glucose transporter alters multiple regulatory steps in glycogen metabolism. Mol Cell Biol 2005;25:9713-23.

13. Ahmed-Sorour H, Bailey CJ. Role of ovarian hormones in the long-term control of glucose homeostasis, glycogen formation and gluconeogenesis. Ann Nutr Metab 1981;25:208-12.

14. Campbell SE, Febbraio MA. Effect of the ovarian hormones on GLUT4 expression and contraction-stimulated glucose uptake. Am J Physiol Endocrinol Metab 2002;282:E1139-46.

15. Stenbit AE, Burcelin R, Katz EB, Tsao TS, Gautier N, Charron MJ, et al. Diverse effects of Glut 4 ablation on glucose uptake and glycogen synthesis in red and white skeletal muscle. J Clin Invest 1996;98:629-34.

16. Butcher RL, Collins WE, Fugo NW. Plasma concentration of LH, $\mathrm{FSH}$, prolactin, progesterone and estradiol-17beta throughout the 4-day estrous cycle of the rat. Endocrinology 1974;94:1704-8.

17. Goldman JM, Murr AS, Cooper RL. The rodent estrous cycle: characterization of vaginal cytology and its utility in toxicological studies. Birth Defects Res B Dev Reprod Toxicol 2007;80:84-97.

18. Olson ME, Bruce J. Ovariectomy, ovariohysterectomy and orchidectomy in rodents and rabbits. Can Vet J 1986;27:523-7.

19. Martinez-Gomez M, Chirino R, Beyer C, Komisaruk BR, Pacheco P. Visceral and postural reflexes evoked by genital stimulation in urethane-anesthetized female rats. Brain Res 1992;575:279-84.

20. Zepeda RC, Barrera I, Castelán F, Soto-Cid A, Hernández-Kelly LC, López-Bayghen E, et al. Glutamate-dependent transcriptional regulation in bergmann glia cells: involvement of p38 MAP kinase. Neurochem Res 2008;33:1277-85.

21. Hernández-Aragón LG, García-Villamar V, Carrasco-Ruiz ML, Nicolás-Toledo L, Ortega A, Cuevas-Romero E, et al. Role of estrogens in the size of neuronal somata of paravaginal ganglia in ovariectomized rabbits. Biomed Res Int 2017;2017:2089645.

22. Passonneau JV, Lauderdale VR. A comparison of three methods of glycogen measurement in tissues. Anal Biochem 1974;60:405-12.

23. Morimoto S, Cerbón MA, Alvarez-Alvarez A, Romero-Navarro G, Díaz-Sánchez V. Insulin gene expression pattern in rat pancreas during the estrous cycle. Life Sci 2001;68:2979-85.

24. Kelly DM, Akhtar S, Sellers DJ, Muraleedharan V, Channer K, Jones $\mathrm{TH}$. Testosterone differentially regulates targets of lipid and glucose metabolism in liver, muscle and adipose tissues of the testicular feminised mouse. Endocrine 2016;54:504-15.

25. Barros RP, Gabbi C, Morani A, Warner M, Gustafsson JA. Participation of ERalpha and ERbeta in glucose homeostasis in skeletal muscle and white adipose tissue. Am J Physiol Endocrinol Metab 2009;297:E124-33.

26. Rüegg J, Cai W, Karimi M, Kiss NB, Swedenborg E, Larsson C, et al. Epigenetic regulation of glucose transporter 4 by estrogen receptor $\beta$. Mol Endocrinol 2011;25:2017-28.

27. Puah JA, Bailey CJ. Effect of ovarian hormones on glucose metabolism in mouse soleus muscle. Endocrinology 1985;117:1336-40. 